\title{
Correction to: Enhancing Statistical Multiple Sequence Alignment and Tree Inference Using Structural Information
}

\author{
Joseph L. Herman
}

Correction to:

Chapter 10 in: Tobias Sikosek (ed.),

Computational Methods in Protein Evolution,

Methods in Molecular Biology, vol. 1851,

https://doi.org/10.1007/978-1-4939-8736-8_10

The published version of this book included errors in code listings in Chapter 10. These code listings have been corrected and text has been updated.

The updated online version of this chapter can be found at

https://doi.org/10.1007/978-1-4939-8736-8_10 